\title{
Thymic activity in immune recovery after allogeneic hematopoietic stem cell transplantation in children
}

\author{
MAEGORZATA JANECZKO-CZARNECKA, BLANKA RYBKA, RENATA RYCZAN-KRAWCZYK, \\ KRZYSZTOF KAEWAK, MAREK USSOWICZ
}

Department of Pediatric Hematology, Oncology and Bone Marrow Transplantation, Wroclaw Medical University, Wroclaw, Poland

\begin{abstract}
Thymic output was studied prospectively in 52 children who underwent allogeneic hematopoietic stem cell transplantation (allo-HSCT). Thymic activity was assessed by quantification of recent thymic emigrants (RTE) discriminated from the rest of naive T cells by immunophenotype CD3+/CD4+/ $C D 31+/ C D 45 R A+$. Thymic output was analyzed in correlation with the kinetics of immune recovery and in relation to other potential risk factors that may influence thymopoiesis: underlying disease, type of HSCT, source of stem cells, age of recipient and donor, type of conditioning, implemented graft versus host disease $(G v H D)$ prophylaxis, viral reactivations (herpes viruses cytomegalovirus - CMV, Epstein-Barr virus - EBV, adenovirus - ADV, BK virus - BKV), occurrence and grade of both acute and chronic graft versus host disease $(a G v H D, c G v H D)$ and number of transplanted CD34 cells/kg. The absolute count of RTE in peripheral blood was evaluated at 6 time points: before the conditioning and on days $+15,+30,+60,+90$ and +180 after HSCT. Occurrence of grade II-IV aGvHD was the most important factor associated with low RTE counts after HSCT. History of malignant disease, and transplantation from matched unrelated donor were risk factors for lower thymic output. We found a weak inverse correlation between the age of the recipient and thymic output on post-HSCT day +180 . Source of stem cells, type of conditioning, viral reactivations, occurrence of chronic GvHD, age of the donor and the number of transplanted CD34 cells/kg did not affect thymopoiesis in our study group. These preliminary findings and identification of risk factors for deterioration of thymic activity may in the future help in selecting candidates for thymus rejuvenation strategies.
\end{abstract}

Key words: thymic activity, immune recovery, recent thymic emigrants, thymopoiesis, pediatric allogeneic hematopoietic stem cell transplantation, graft versus host disease.

(Cent Eur J Immunol 2020; 45 (2): 151-159)

\section{Introduction}

Allogeneic hematopoietic stem cell transplantation (allo-HSCT) is an established method used in the treatment of malignant and non-malignant diseases. The procedure is followed by a period of profound lymphopenia and immunodeficiency as a consequence of immune system ablation with pre-transplant conditioning and post-transplant immunosuppressive therapy. The incidence of severe post-transplant infections, relapses or even secondary malignancies can be directly related to inadequate immune recovery [1, 2]. Proper T-cell function has been shown to be crucial in determining the HSCT outcome. There is growing evidence that adequate thymopoiesis is essential for immune reconstitution, de novo generation of T cells after HSCT and functional recovery of the immune system [3, 4].
T-cells can be generated by two different routes: the thymus-independent pathway predominates in the early post-transplant period while active thymopoiesis (thymus-dependent pathway) takes over in the late post-transplant period and results in reconstitution of a functionally competent, fully mature, naive T-cell compartment with broad antigenic specificity $[5,6]$. The thymus-independent pathway originates from the transfer of mature, graft-derived donor $\mathrm{T}$ cells or residual recipient T-cells that survived pre-transplant conditioning. These T-cell populations undergo peripheral expansion in response to lymphopenia and high cytokine levels or as a result of an interaction with a known antigen. They respond quickly to previously encountered pathogens such as herpes viruses cytomegalovirus (CMV) or Epstein-Barr virus (EBV), are

Correspondence: Małgorzata Janeczko-Czarnecka MD, Department of Pediatric Hematology, Oncology and Bone Marrow Transplantation, Wroclaw Medical University, 213 Borowska St., 50-556 Wroclaw, Poland, e-mail: ml.janeczko@gmail.com Submitted: 20.01.2019; Accepted: 15.05.2019 
easier to trigger and faster to respond but they have limited T-cell receptor (TCR) diversity, dependent on the number of T-cell precursors in the graft [7]. Thymus independent peripheral T-cell expansion results in late effector memory T-cell skewing and contraction of the T-cell repertoire diversity, and leads to impaired immunologic responses to new antigens. The recovery of T-cell counts during the first 6 months after transplantation depends mainly on the cytokine-driven (interleukin [IL]-15, IL- 2, IL-7) peripheral expansion of mature T-cells [3]. Whereas peripheral proliferation of T-cells protects the host against infections, de novo generation and differentiation of donor-derived

Table 1. Characteristics of the study group

\begin{tabular}{|c|c|c|}
\hline Patients & $n$ & $\%$ \\
\hline \multicolumn{3}{|l|}{ Sex } \\
\hline Male & 35 & 67 \\
\hline Female & 17 & 33 \\
\hline \multicolumn{3}{|l|}{ Type of donor } \\
\hline Matched unrelated (MUD) & 40 & 77 \\
\hline Matched family (MFD) & 12 & 23 \\
\hline \multicolumn{3}{|l|}{ Source of stem cells } \\
\hline Peripheral blood (PB) & 42 & 81 \\
\hline Bone marrow (BM) & 7 & 13 \\
\hline Cord blood (CB) & 3 & 6 \\
\hline \multicolumn{3}{|l|}{ Diagnosis } \\
\hline Malignant & 30 & 58 \\
\hline Acute lymphoblastic leukemia (ALL) & 14 & 27 \\
\hline Acute myeloblastic leukemia (AML) & 8 & 15 \\
\hline Myelodysplastic syndrome (MDS) & 3 & 6 \\
\hline Hodgkin lymphoma (HL) & 2 & 4 \\
\hline Chronic myeloid leukemia (CML) & 1 & 2 \\
\hline Anaplastic large cell lymphoma (ALCL) & 1 & 2 \\
\hline Osteomyelofibrosis & 1 & 2 \\
\hline Non-malignant & 22 & 42 \\
\hline Severe aplastic anemia (SAA) & 5 & 9 \\
\hline Metabolic diseases & 6 & 12 \\
\hline Immune deficiencies (ID*) & 12 & 21 \\
\hline \multicolumn{3}{|l|}{ Conditioning } \\
\hline Reduced-intensity conditioning (RIC) & 26 & 50 \\
\hline Myeloablative (MA) & 21 & 40 \\
\hline Non-myeloablative (NMA) & 5 & 10 \\
\hline \multicolumn{3}{|l|}{ Graft versus host disease prophylaxis } \\
\hline Anti-thymocyte globulin (ATG) & 42 & 81 \\
\hline Without ATG & 10 & 19 \\
\hline
\end{tabular}

lymphoid-myeloid progenitors (LMPs) within the recipient thymus is required for durable, effective, and flexible immunity that can respond to a broad range of pathogens encountered by the host and to tumor antigens. In humans, the production of naive $\mathrm{T}$ cells via the second pathway is dependent on a functional thymus, because in athymic patients (i.e. complete DiGeorge syndrome or patients thymectomized before HSCT) no naive T-cells are detectable after transplantation [8]. LMPs migrate from the bone marrow via the circulation and repopulate the thymus with thymocyte precursors that can reconstitute thymopoiesis. The thymus provides the essential microenvironment (stroma) that supports T-cell proliferation, selection, and differentiation into RTEs. In the thymus-dependent pathway RTEs are generated, which is crucial for a durable $\mathrm{T}$-cell reconstitution and a diverse T-cell receptor (TCR) repertoire, capable of responding to a great spectrum of antigens $[9,10]$.

The analysis of thymic reconstitution can be useful for predicting clinical outcomes of transplantation including opportunistic infections, disease relapse or overall survival [11]. The most reliable method of thymic function assessment is still under debate. The quantification of RTEs with flow cytometry is based on the expression of surface antigens CD45RA and CD31 as phenotypic markers of naive T cells [7, 12, 13]. However, naive CD45RA+T-cells can undergo peripheral expansion without phenotypic changes, especially if not antigen-activated. Furthermore, memory CD45RO+ T-cells may revert to a naive CD45RA+ phenotype, especially in the case of persistent viral infections [6, 14]. Another method for estimation of the thymic output is PCR detection and quantification of signal joint TCR excision circles (sjTRECs) formed during rearrangement of the TCR chain in maturing T-cells. These by-products can be quantified in peripheral blood $\mathrm{T}$ cells as a marker of active thymopoiesis [14]. Both methods are techniques requiring only a small amount of blood and, as many authors have proven, TREC levels correlate with the levels of phenotypically naive $\mathrm{T}$ cells $[15,16]$.

Several factors injuring the thymus can delay the recovery of thymopoiesis after HSCT including: increasing age of the recipient, occurrence and grade of acute or chronic graft versus host disease (aGvHD, cGvHD), intensity of conditioning, source of stem cells, infections in the transplant period (especially CMV reactivation) or previous treatment of the underlying disease (malignant or non-malignant) $[15,17]$.

\section{Material and methods}

The analysis included 52 patients who underwent allo-HSCT in the Department of Pediatric Hematology, Oncology and Bone Marrow Transplantation at Wrocław Medical University between September 2016 and July 2017. Patient characteristics are summarized in Table 1. 
Median age of the patients at the time of HSCT was 7.7 years (range 6 months to 19.5 years). Patients were divided into 3 groups: myeloablative (MA), reduced intensity (RIC) and non-myeloablative (NMA) conditioning according to the criteria described by Bacigalupo et al. [18]. During the observation period 6 patients died (12\%). Causes of death were: progression of the disease $(n=1)$, $\operatorname{aGvHD}(n=1)$ or infections $(n=4)$.

Immune recovery and thymic function were monitored by sampling peripheral blood at 6 time points: before transplantation (day before the conditioning onset) and +15 , $+30,+60,+90,+180$ days after HSCT. Thymopoiesis was assessed by quantification with flow cytometry of an absolute count of RTEs defined as naive T-cells expressing CD3+/CD4+/CD31+/CD45RA+. The absolute value of RTE was calculated based on the percentage ratio of the number of events (T-cells expressing CD3+/CD4+/ $\mathrm{CD} 31+/ \mathrm{CD} 45 \mathrm{RA}+$ ) in the lymphocyte gateway to the total number of events (total T-cell ratio in the gateway). Afterwards this percentage was multiplied by the absolute lymphocyte value in a given blood sample (total leukocytosis $\times \%$ lymphocytes in peripheral blood smear). Absolute value of RTE was defined as the number of cells per microliter of blood.

Lymphocyte immunophenotyping was performed on fresh whole blood samples on EDTA (ethylenediaminetetraacetic acid) and on transplant material samples from blood apheresis or from bone marrow by direct 4- or 6-color immunofluorescence. The following monoclonal antibodies were used: CD45RA-FITC (Beckman Coulter), CD31-PE (Beckman Coulter), CD3 APC-Cy7 (Becton Dickinson), CD4-PerCP-Cy5.5 (Becton Dickinson). Lymphocyte subset percentage and absolute counts were enumerated with BD Multitest 6-Color TBNK (Becton Dickinson): CD3-FITC, CD4-PE-Cy7, CD8-APC-Cy7, CD56-PE, CD16-PE, CD19-APC, CD45-PerCP-Cy (Fig. 1).
Lymphocyte gate purity was equal to at least $98 \%$. Gated lymphocytes (5000) were analyzed with a FACS Canto II flow cytometer using BD FACSC Diva software version 8.0.1 (Becton Dickinson). This study was approved by the Bioethical Committee at Wrocław Medical University.

\section{Statistical methods}

The statistical analysis was performed using STATISTICA 13.3. The influence of parameters such as underlying disease (malignant, non-malignant), source of stem cells (PBSC, BM, CB), type of HSCT (MUD, MFD), type of conditioning (RIC, MA, NMA), GvHD prophylaxis (with/ without ATG), CMV, EBV, ADV and BK virus (BKV) reactivation, occurrence and grade of aGvHD or cGvHD on thymopoiesis after HSCT was analyzed using analysis of variance with repeated measures (ANOVA). The differences in mean values of RTEs at defined time-points between the groups were tested due to the lack of normal distribution with the nonparametric Mann-Whitney $U$ test. The influence of parameters such as age of the recipient, age of the donor, and number of transplanted CD34+ cells $/ \mathrm{kg}$ due to the lack of normal distribution was analyzed with Spearman's rank correlation and Kendall's tau correlation tests.

\section{Results}

Summarized results of RTE median values at defined time points are presented in Table 2. Furthermore, we marked with asterisks $\left(^{*}\right)$ values that represent significant differences between groups.

\section{Type of HSCT}

There was a significant difference in thymopoiesis between patients transplanted from MUD and MFD (ANOVA $p=0.002$ ). In the MFD group the values of RTEs
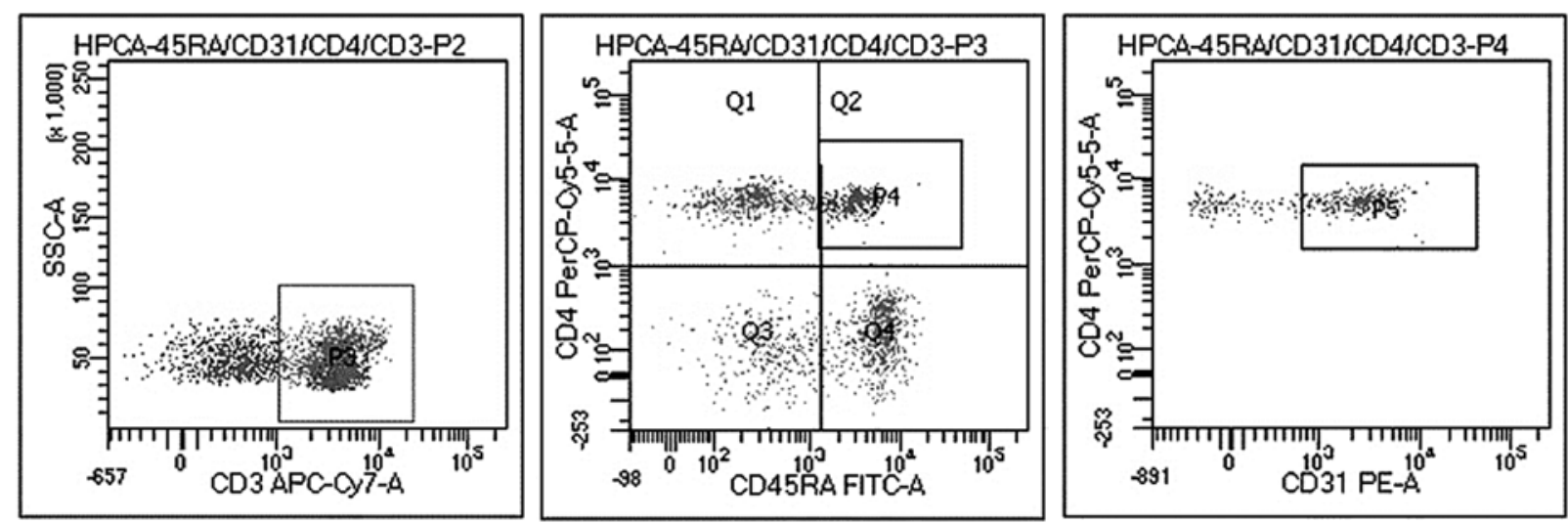

Fig. 1. Gating strategy for CD3+CD4+CD31+CD45RA+. Gating was performed on lymphocytes (purity $>98 \%$ ) using Multitest 6-Color TBNK. The population of CD3+ cells (gate P3) was analyzed for expression of CD45RA+ CD4+ (gate P4). In the next step the population of CD3+CD4+CD45RA+ was analyzed for expression of CD31 (gate P5) 
Table 2. Median values of RTEs at defined time points (lymphocyte count: cells/ $\mu$ l). Asterisks represent significant differences between groups $\left({ }^{*} p<0.05\right)$

\begin{tabular}{|c|c|c|c|c|c|c|}
\hline Parameter & pre-HSCT & day +15 & day +30 & day +60 & day +90 & day +180 \\
\hline \multicolumn{7}{|l|}{ Type of donor } \\
\hline MUD & 428.4 & $19.5^{*}$ & $11.5^{*}$ & $9.0^{*}$ & $10.5^{*}$ & $68.5^{*}$ \\
\hline MFD & 149.4 & $84.3^{*}$ & $338.9^{*}$ & $73.8^{*}$ & $133.4^{*}$ & $173.6^{*}$ \\
\hline \multicolumn{7}{|l|}{ Source of stem cells } \\
\hline $\mathrm{PB}$ & 388.7 & 40.4 & 102.5 & 24.4 & 35.5 & 85.2 \\
\hline BM & 191.5 & 12.5 & 31.4 & 25.8 & 51.9 & 134.8 \\
\hline $\mathrm{CB}$ & 408.7 & 1.7 & 1.6 & 13.3 & 1.0 & 23.4 \\
\hline \multicolumn{7}{|l|}{ Diagnosis } \\
\hline Malignant & $164.6^{*}$ & 36.8 & $145.9^{*}$ & 34.3 & $54.6^{*}$ & 107.1 \\
\hline Non-malignant & $634.0^{*}$ & 31.2 & $6.9^{*}$ & 9.8 & $10.1^{*}$ & 58.9 \\
\hline \multicolumn{7}{|c|}{ Conditioning regimen } \\
\hline RIC & 327.0 & 29.9 & 21.3 & 13.7 & 17.9 & 103.4 \\
\hline MA & 345.7 & 46.1 & 188.5 & 39.5 & 67.1 & 84.5 \\
\hline NMA & 632.2 & 9.1 & 5.7 & 11.8 & 5.3 & 23.5 \\
\hline \multicolumn{7}{|l|}{ ATG } \\
\hline Yes & 447.5 & $16.4^{*}$ & $14.7^{*}$ & $11.8^{*}$ & $16.9^{*}$ & 70.7 \\
\hline No & 75.3 & $97.7^{*}$ & $391.0^{*}$ & $76.0^{*}$ & $133.1^{*}$ & 177.6 \\
\hline \multicolumn{7}{|l|}{ Viral reactivation } \\
\hline CMV (+) & 591.7 & 54.0 & 23.2 & 20.9 & 38.4 & 53.3 \\
\hline CMV (-) & 266.2 & 21.8 & 120.9 & 25.5 & 34.8 & 103.1 \\
\hline $\operatorname{EBV}(+)$ & 767.8 & 4.8 & 8.3 & 1.3 & 3.5 & 86.0 \\
\hline $\operatorname{EBV}(-)$ & 353.8 & 35.5 & 33.0 & 25.8 & 39.0 & 88.3 \\
\hline $\operatorname{ADV}(+)$ & 292.0 & 9.8 & 28.2 & 22.4 & 40.7 & 45.4 \\
\hline $\operatorname{ADV}(-)$ & 396.1 & 37.5 & 97.8 & 24.2 & 35.2 & 95.0 \\
\hline $\mathrm{BKV}(+)$ & 355.4 & 19.7 & 49.4 & 27.1 & 51.0 & 49.7 \\
\hline BKV (-) & 382.4 & 34.8 & 104.6 & 23.5 & 33.9 & 94.3 \\
\hline \multicolumn{7}{|l|}{ aGvHD } \\
\hline Yes & 458.8 & 33.9 & $19.9^{*}$ & 18.1 & $25.9^{*}$ & $45.7^{*}$ \\
\hline No & 253.3 & 31.4 & $186.2^{*}$ & 33.3 & $52.6^{*}$ & $152.9^{*}$ \\
\hline \multicolumn{7}{|l|}{ aGvHD grade } \\
\hline $0-\mathrm{I}$ & 315.2 & 25.2 & $128.7^{*}$ & 25.7 & 35.6 & $102.6^{*}$ \\
\hline II-IV & 489.3 & 46.3 & $14.8^{*}$ & 22.1 & 38.8 & $63.6^{*}$ \\
\hline \multicolumn{7}{|l|}{ cGvHD } \\
\hline Yes & 333.8 & 2.8 & 2.7 & 8.3 & 6.8 & 32.2 \\
\hline No & 463.7 & 18.4 & 16.3 & 12.3 & 18.1 & 76.9 \\
\hline
\end{tabular}




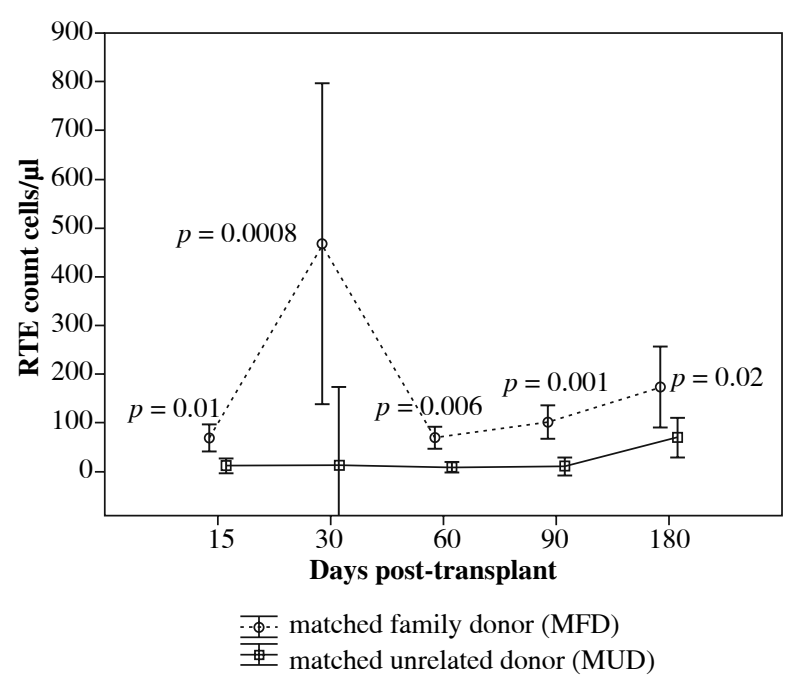

Fig. 2. Reconstitution of RTEs after allogenic HSCT according to type of the transplant (MFD, MUD). Dots represent mean values of RTEs while whiskers represent standard deviation (SD)

were significantly higher on days $+15(p=0.001),+30$ $(p=0.0008),+60(p=0.006),+90(p=0.001)$ and +180 $(p=0.02)$ after allo-HSCT (Fig. 2).

\section{GvHD prophylaxis}

The thymic output was inferior in the patients who received ATG (ANOVA $p=0.000667)$. Significant differences were observed on post-HSCT days $+15(p=0.006),+30$ $(p=0.0002),+60(p=0.0009)$ and $+90(p=0.001)$ (Fig. 3).

\section{Source of stem cells}

The source of stem cells (BM, PBSC or CB) showed no significant impact on the thymic output.

\section{Underlying disease}

Patients transplanted due to malignant diseases showed significant differences in the production of RTEs after allo-HSCT compared with non-malignant patients. A significant difference was observed before conditioning $(p=0.00036)$, on day $+30(p=0.0063)$ and on day +90 $(p=0.039)$ after transplant. It is worth noting that the group with hematological malignancies had lower pre-transplant RTE counts than children with non-malignant diseases but on days +30 and +90 statistically higher values of RTEs were observed than in the group with non-malignant disorders (Fig. 4).

\section{Type of conditioning}

There was no significant difference in RTE counts between the MA, NMA or RIC conditioning groups. Imple-

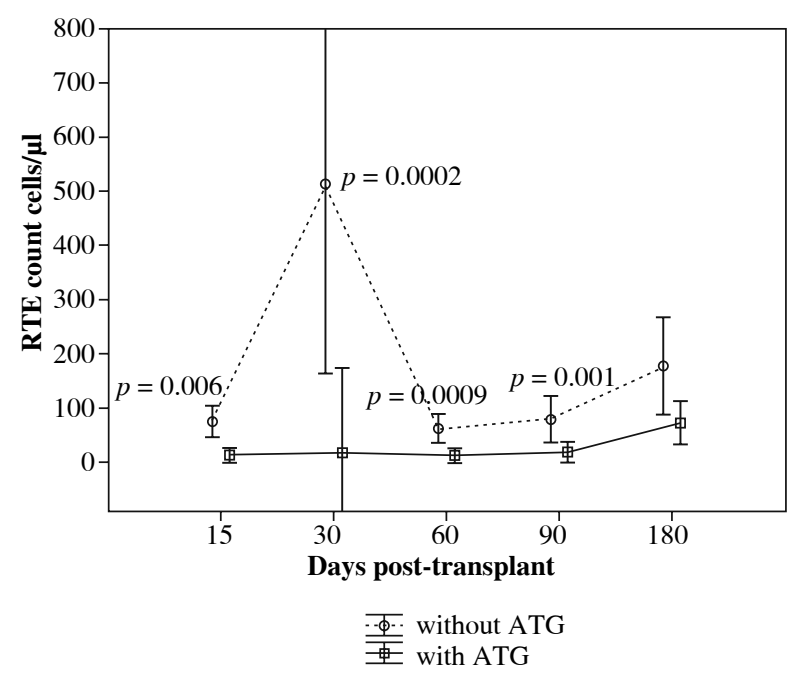

Fig. 3. Reconstitution of RTEs after allogenic HSCT according GvHD prophylaxis (with vs. without ATG). Dots represent mean values of RTEs while whiskers represent standard deviation (SD)

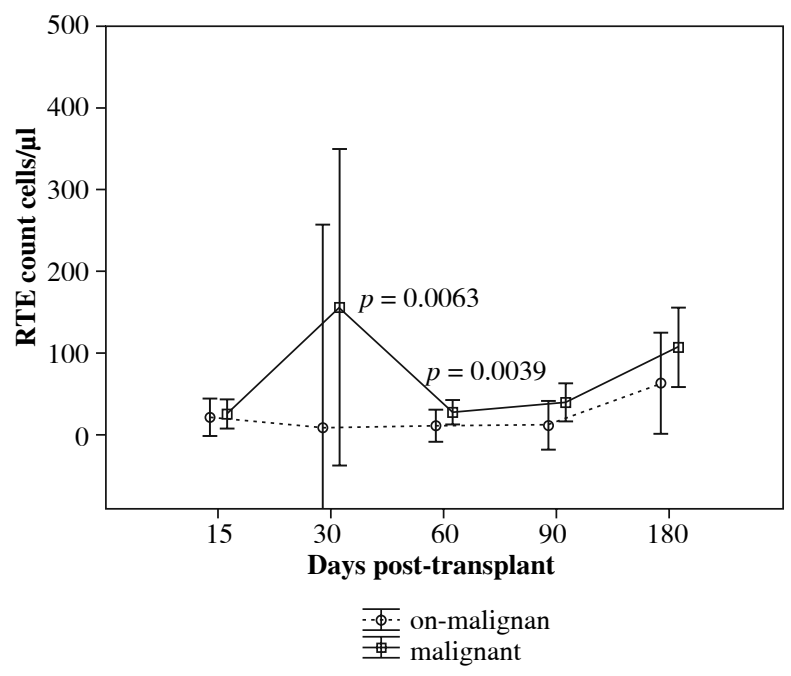

Fig. 4. Reconstitution of RTEs after allogenic HSCT with reference to underlying disease (malignant or non-malignant). Dots represent mean values of RTEs while whiskers represent standard deviation (SD)

mentation of the total body irradiation (TBI) was analyzed separately and was found not to have a significant impact on thymopoiesis.

\section{Viral reactivations}

None of the analyzed viral reactivations (CMV, EBV, $\mathrm{ADV}$ and $\mathrm{BKV}$ ) had a significant impact on thymopoiesis measured by quantification of RTEs. 


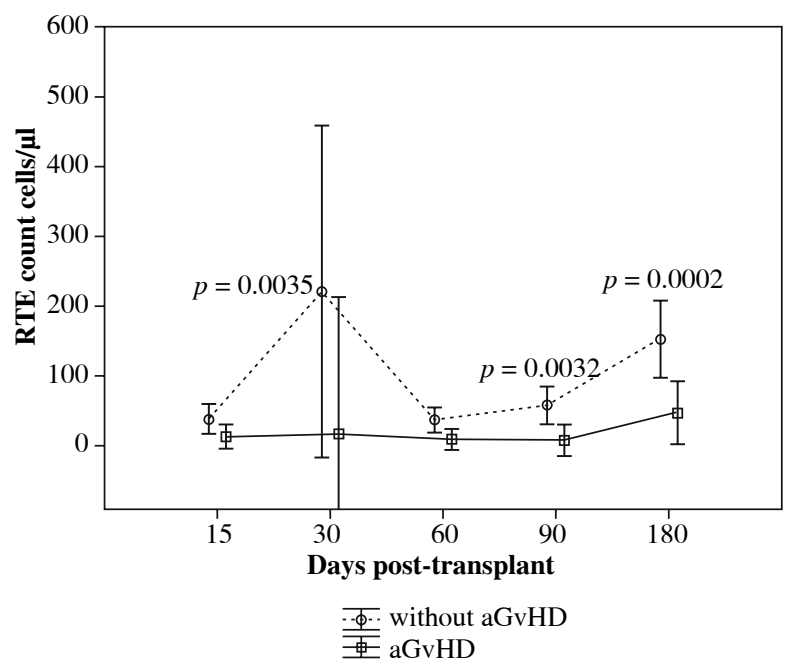

Fig. 5. Reconstitution of RTEs after allogenic HSCT according to occurrence of aGvHD (with vs. without $\mathrm{aGvHD}$ ). Dots represent mean values of RTEs while whiskers represent standard deviation (SD)

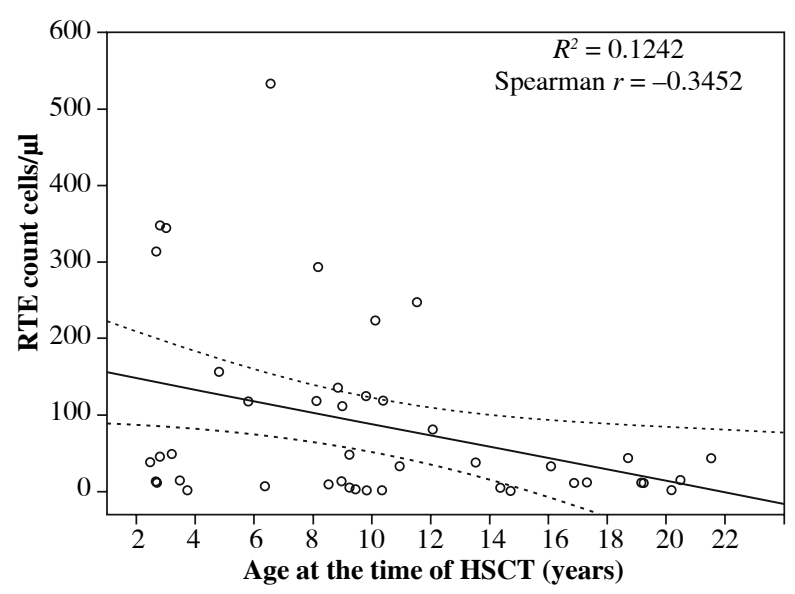

Fig. 7. Reconstitution of RTEs after allogenic HSCT at post-transplant day +180 in accordance to the age of the recipient

\section{Graft versus host disease}

During the observation period, 31 patients developed acute GvHD. The upfront therapy consisted of methylprednisolone at a dose of 1 to $2 \mathrm{mg} / \mathrm{kg} \mathrm{BW}$ and was continued until a clinical response was seen. Two patients did not respond to the steroid therapy within 7 days and therapy with monoclonal antibodies (etanercept, basiliximab) and extracorporeal photopheresis was required. A significantly lower thymic activity was observed in the group with aGvHD $(n=31)$ compared to patients without aGvHD $(n=21), p=0.003$. The difference was noted on days +30

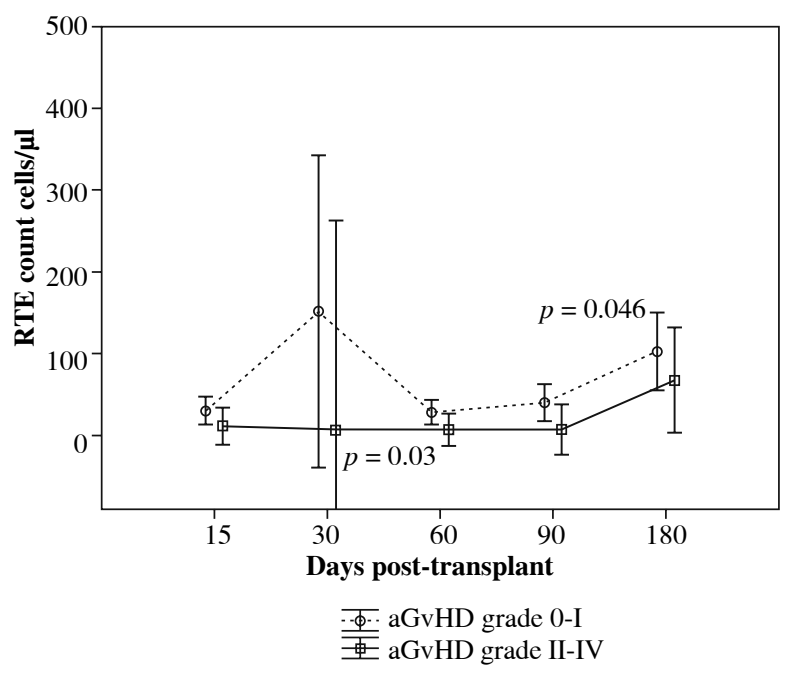

Fig. 6. Reconstitution of RTEs after allogenic HSCT according to grade of aGvHD (grade 0-I vs. grade II-IV). Dots represent mean values of RTEs while whiskers represent standard deviation (SD)

$(p=0.035),+90(p=0.032)$ and $+180(p=0.0002)$ after HSCT (Fig. 5). A significant difference in values of RTEs between groups with grade 0 -I and grade II-IV aGvHD was seen $(p=0.002)$. The RTE counts were significantly lower in the group with aGvHD grade II-IV on days +30 $(p=0.03)$ and $+180(p=0.046)$ (Fig. 6). In patients without aGvHD history, significantly higher thymic output was observed in the MFD group. The difference was observed on days $+15(p=0.015),+30(p=0.0031),+60$ $(p=0.0074)$ and $+90(p=0.014)$.

There was no significant difference in values of RTEs between the groups with or without cGvHD.

\section{Age of the recipient}

A weak negative correlation between the age of the recipient and values of RTE on day +180 was found $(p=0.02)$. The line of best fit was calculated as $Y$ (value of $R T E)=147.9189-7.4873 * X$ (age of the recipient) $($ Fig. 7$)$.

\section{Age of the donor}

No correlation was found between the age of the donor and values of RTEs.

\section{Number of transplanted stem cells}

The number of transplanted CD34 cells/kg showed no impact on the post-transplant thymus output.

\section{Discussion}

Our study showed the impact of the type of allo-HSCT (MUD, MFD), administration of anti-thymocyte globulin, 
underlying disease (malignant or non-malignant), occurrence and grade of aGvHD and age of the recipient on the post-transplant values of RTEs and thymopoiesis.

\section{Type of transplant and GvHD prophylaxis}

Patients undergoing MUD transplantations have worse thymic function than patients undergoing MFD transplantations for a period of 6 months after HSCT. A study by Small et al. proved that HSCT from MUDs were associated with lower numbers of CD45RA+ cells after transplantation compared to patients receiving grafts from matched sibling donors (MSD) [13]. Weinberg et al. found that compared with unrelated donor grafts, recipients of matched sibling grafts had higher TREC levels but this effect disappeared if a history of GvHD was taken into account [15]. The authors concluded that the lower TREC levels in the recipients of MUD-HSCT resulted from their increased incidence of GvHD. In our study, no differences in RTE counts between MUD and MFD HSCT subgroups were observed in the patients who developed aGvHD, but in children without aGvHD MFD-HSCT recipients showed higher thymic output. These observations strongly point to the role of pre-transplant immunosuppressive prophylaxis, which was lower in the MFD HSCT, and to the effect of immunosuppression in symptomatic GvHD patients.

The administration of ATG as GvHD prophylaxis impaired thymic function for three months after transplantation, when the mean values of RTEs equalized in both groups. ATG was administered in $97 \%$ of MUD-HSCT and $25 \%$ of MFD-HSCT and was responsible for a delay in thymic recovery.

Thymopoiesis can be affected by the type of conditioning preceding HSCT, as the thymus is a sensitive target for intensive chemo- and radiotherapy $[8,19]$. However, in our group no significant differences in RTE counts between the groups receiving MA, RIC or NMA conditioning were observed and TBI did not affect the thymopoiesis either.

\section{Underlying disease}

Diagnosis and pre-transplantation therapy can have an impact on thymopoiesis as seen in the patients with hematological malignancies who had lower pre-transplant thymic function. These findings are in accordance with the observations published by Clave, Lisini et al., who described a group of 57 patients transplanted for either malignant or non-malignant diseases, and before transplantation TREC values were significantly lower in the 46 patients with hematological malignancies than in the 11 with non-malignant disorders [20]. Other studies confirmed that hematologic malignancies and chemotherapy are important and independent risk factors related to thymic inhibition [21-23]. Petridou et al. published a study of 120 patients (30 with T-cell leukemia/non-Hodgkin's lymphoma, 30 age- and sexmatched with B-cell ALL and 60 healthy hospital controls) and reported that in a group with T-cell malignancies a reduction of TREC values was noted whereas in children with B-cell ALL only slightly and non-significantly lower TREC values were observed compared to healthy children controls [24]. This observation was not confirmed by our study, but further studies on larger homogeneous populations may help elucidate this finding.

\section{Acute graft versus host disease}

The thymus could be a very sensitive target for the GvHD but in children and young adults can recover its function. We confirmed that not only occurrence of aGvHD significantly impaired thymic output, but also higher grades (II-IV) were related to lower RTE values. A comparison between steroid-responsive and steroid-resistant patients was not possible due to the low number of events. Similar results were described by Clave et al. in a group of 93 patients where thymic activity was measured by both sjTREC real-time PCR and flow cytometry (CD4+CD45RA+CD62L+) [9]. Absolute sjTREC number was lower at 6 months in patients with aGvHD and associated with lower absolute counts of naive T-lymphocytes at 6 and 12 months, which is in line with our observations. Eyrich et al. performed an analysis of the thymic activity in a group of 164 children with a median follow-up of 333 days (range 50-1105) and found that acute (grade II-IV) and chronic (limited and extensive) GvHD significantly impaired thymic output, resulting in lower TREC levels in patients with GvHD [25]. In our study group we did not observe any significant correlation between occurrence of cGvHD and thymic function due to the observation period being too short (until 180 days after transplantation). Another study, by Hazenberg et al., described significantly lower thymic output caused by aGvHD and/or infectious complications measured by TREC quantification, but the authors suggested that increased TREC level is a composite of thymic output of TREC T cells, accumulation of these cells in the periphery over time, and death of these cells [26]. In Weinberg's study low TRECs after HSCT correlated with the presence or history of GvHD [15]. Ringhoffer et al. concluded that aGvHD was the most prominent negative factor by performing univariate analysis and in Saglio's study the only factor influencing thymus activity after transplantation was GvHD $[27,28]$. However, the detrimental effect of GvHD on the thymic activity can be a consequence of the immunosuppressive therapy that was administered, as Chen et al. described in their paper [29]. Although impaired thymic output is associated with GvHD, the mechanisms are complex and need to be elucidated.

\section{Age of the recipient}

Aging is believed to be a major factor affecting the function of the thymus. Thymic involution begins after birth, with a decrease of $3 \%$ per year, and is present until at least the fifth decade of life. The superior HSCT outcome 
in younger patients suggests a role of the thymus function in preventing post-transplant events [30]. Eyrich et al. performed a multivariate analysis in a group of 164 children by measuring TRECs and spontaneous expression of Ki67 in peripheral T-cell subsets and observed a negative correlation between the onset of thymic recovery and the recipient age [31]. A few other studies proved that younger patients achieve faster immune recovery than older ones $[5,9,14]$. We also observed a weak negative correlation between the values of RTEs and the age of the recipient, but the effect took place no sooner than 6 months after transplantation.

\section{Viral reactivations}

The thymus is a sensitive target for reactivations of viral infections (CMV, EBV, ADV, BKV), which lead to acute involution and impaired function of this organ [32]. CMV directly invades the thymus, causing disruption of its architecture, degradation of the thymic microenvironment and poor immune recovery after HSCT [33]. Toubert et al. and Klaus da Rocha et al. found an association between poor thymic recovery and a higher incidence of CMV reactivation in allo-HSCT patients [14, 23]. Also results published by Lewin et al. showed that low TREC levels were significantly correlated with severe opportunistic infections [34]. Furthermore, Brown JA et al. and Politikos et al. reported that clearance of CMV viremia significantly correlated with the recovery of naive T-cells and reconstitution of thymopoiesis [5, 35]. In our study however none of the analyzed viral reactivations (CMV, EBV, ADV and $\mathrm{BKV}$ ) had a significant impact on thymopoiesis measured by quantification of RTEs. However, these results may be related to the small study size.

\section{Thymus rejuvenation strategies}

Identification of risk factors for the deterioration of thymic activity may help in the future in selecting candidates for thymus rejuvenation strategies that are presently studied [36]. Current clinical trials in adult patients after HSCT investigate keratinocyte growth factor (KGF), cytokines (IL-7 and IL-22), and hormonal modulation including sex steroid inhibition and growth hormone administration. In younger patients these therapies could speed up the immune recovery process and thus reduce the number of post-transplant complications resulting from delayed immune reconstitution [36]. In animal studies caloric restrictions enhanced thymopoiesis and protected mice from age-related thymic involution and increased the proportion of nadve to memory T-cells in the peripheral blood [37]. In contrast, a high-fat diet had a negative impact on thymic output in another study in mice [38]. This strategy is however controversial and some authors showed that it can in fact impair thymic recovery, and especially hinder NK maturation and function. Therefore this therapeutic option needs further investigation and findings should be interpreted with caution.

\section{Conclusions}

This study showed that the thymic output can be evaluated using an economical and fast flow-cytometric method. Analysis showed that pre-transplant conditions and physiological phenomena affect the post-transplant immune recovery. Lower age at transplantation showed an inverse correlation with RTE counts, confirming that age-related thymic involution has an impact on post-transplant recovery. Pre-transplant thymic injury, and administration of lymphotoxic ATG additionally affected the thymic output by decreased RTE production. Given the small study size and heterogeneity of the analyzed patients, these results should be further confirmed in other population groups. These preliminary findings and identification of the risk factors for the deterioration of thymic activity may in the future help in selecting candidates for thymus rejuvenation strategies.

\section{The authors declare no conflict of interest.}

\section{References}

1. Wils E, van der Holt B, Broers AEC, et al. (2011): Insufficient recovery of thymopoiesis predicts for opportunistic infections in allogeneic hematopoietic stem cell transplant recipients. Haematologica 96: 1846-1854.

2. Mohty B, Mohty M (2011): Long-term complications and side effects after allogeneic hematopoietic stem cell transplantation: An update. Blood Cancer J 1: e16.

3. Roux E, Dumont-Girard F, Starobinski M, et al. (2000): Recovery of immune reactivity after T-cell-depleted bone marrow transplantation depends on thymic activity. Blood 96 : 2299-2303.

4. Berzins SP, Boyd RL, Miller JFAP (1998): The Role of the Thymus and Recent Thymic Migrants in the Maintenance of the Adult Peripheral Lymphocyte Pool. J Exp Med 187: 1839-1848.

5. Politikos I, Boussiotis VA (2014): The role of the thymus in T-cell immune reconstitution after umbilical cord blood transplantation. Blood 124: 3201-3211.

6. Williams K, Hakim F, Gress R (2008): T-cell reconstitution following lymphodepletion. Semin Immunol 22: 629.

7. Głowala-Kosińska M, Chwieduk A, Smagur A, et al. (2016): Thymic Activity and T Cell Repertoire Recovery after Autologous Hematopoietic Stem Cell Transplantation Preceded by Myeloablative Radiotherapy or Chemotherapy. Biol Blood Marrow Transplant 22: 834-842.

8. Heither A, Neu N, Kern H, et al. (1997): Essential role of the thymus to reconstitute naive (CD45RA+) T-helper cells after human allogeneic bone marrow transplantation. Blood 90: 850-857.

9. Clave E, Busson M, Douay C, et al. (2009): Acute graft-versus-host disease transiently impairs thymic output in young patients after allogeneic hematopoietic stem cell transplantation. Blood 113: 6477-6484. 
10. Mackall C, Granger L, Sheard M, et al. (1993): T-cell Regeneration After Bone Marrow Transplantation: Differential CD45 Isoform Expression on Thymic-Derived Versus Thymic-Independent Progeny. Blood 82: 2585-2595.

11. Bains I, Antia R, Callard R, Yates AJ (2012): Quantifying the development of the peripheral naive CD4 + T-cell pool in humans. Blood 113: 5480-5487

12. Mackall CL, Fleisher TA, Brown MR, et al. (1995): Age, Thymopoiesis, and CD4+ T-Lymphocyte Regeneration after Intensive Chemotherapy. N Engl J Med 332: 143-149.

13. Small TN, Papadopoulos EB, Boulad F, et al. (1999): Comparison of immune reconstitution after unrelated and related T-cell-depleted bone marrow transplantation: Effect of patient age and donor leukocyte infusions. Blood 93: 467-480.

14. Toubert A, Glauzy S, Douay C, Clave E (2012): Thymus and immune reconstitution after allogeneic hematopoietic stem cell transplantation in humans: Never say never again. Tissue Antigens 79: 83-89.

15. Weinberg K, Blazar BR, Wagner JE, et al. (2001): Factors affecting thymic function after allogeneic hematopoietic stem cell transplantation. Blood 97: 1458-1466.

16. McFarland RD, Douek DC, Koup RA, Picker LJ (2000): Identification of a human recent thymic emigrant phenotype. Proc Natl Acad Sci 97: 4215-4220.

17. Gaballa A, Sundin M, Stikvoort A, et al. (2016): T cell receptor excision circle (TREC) monitoring after allogeneic stem cell transplantation; a predictive marker for complications and clinical outcome. Int J Mol Sci 17: 1-17.

18. Bacigalupo A, Ballen K, Rizzo D, et al. (2009): Defining the intensity of conditioning regimens. Biol Blood Marrow Transplant 15: 1628-1633.

19. Jiménez M, Martínez C, Ercilla G, et al. (2005): Reducedintensity conditioning regimen preserves thymic function in the early period after hematopoietic stem cell transplantation. Exp Hematol 33: 1240-1248.

20. Clave E, Lisini D, Douay C, et al. (2013): Thymic function recovery after unrelated donor cord blood or T-cell depleted HLA-haploidentical stem cell transplantation correlates with leukemia relapse. Front Immunol 4: 1-8.

21. Clave E, Rocha V, Talvensaari K, et al. (2005): Prognostic value of pretransplantation host thymic function in HLA-identical sibling hematopoietic stem cell transplantation. Blood 105: 2608-2613.

22. Chen X, Barfield R, Benaim E, et al. (2005): Prediction of $\mathrm{T}$-cell reconstitution by assessment of $\mathrm{T}$-cell receptor excision circle before allogeneic hematopoietic stem cell transplantation in pediatric patients. Blood 105: 886-893.

23. da Rocha LKA, de Barros SF, Bandeira F, et al. (2018): Thymopoiesis in pre-and post-hematopoietic stem cell transplantation. Front Immunol 9: 1-13.

24. Petridou E, Klimentopoulou AE, Moustaki M, et al. (2005): Recent thymic emigrants and prognosis in T- and B-cell childhood hematopoietic malignancies. Int J Cancer 101: 74-77.

25. Eyrich M, Wollny G, Tzaribaschev N, et al. (2005): Onset of thymic recovery and plateau of thymic output are differentially regulated after stem cell transplantation in children. Biol Blood Marrow Transplant 11: 194-205.

26. Hazenberg MD, Otto SA, De Pauw ES, et al. (2002): T-cell receptor excision circle and T-cell dynamics after allogeneic stem cell transplantation are related to clinical events. Blood 99: 3449-3453.
27. Ringhoffer S, Rojewski M, Döhner H, et al. (2013): T-cell reconstitution after allogeneic stem cell transplantation: Assessment by measurement of the sjTREC/ $\beta$ TREC ratio and thymic nadve T cells. Haematologica 98: 1600-1608.

28. Saglio F, Cena S, Berger M, et al. (2015): Association between Thymic Function and Allogeneic Hematopoietic Stem Cell Transplantation Outcome: Results of a Pediatric Study. Biol Blood Marrow Transplant 21: 1099-1105.

29. Chen Y, Qiao S, Tuckermann J, et al. (2010): Thymus-derived glucocorticoids mediate androgen effects on thymocyte homeostasis. FASEB J 24: 5043-5051.

30. Lynch H, Golberg G, Chidgey A, et al. (2009): Thymic involution and immune reconstitution. Trends Immunol 30: 366-373.

31. Eyrich M, Leiler C, Lang P, et al. (2003): A prospective comparison of immune reconstitution in pediatric recipients of positively selected CD34+peripheral blood stem cells from unrelated donors vs recipients of unmanipulated bone marrow from related donors. Bone Marrow Transplant 32: 379-390.

32. Savino W (2006): The thymus is a common target organ in infectious diseases. PLoS Pathog 2: e62.

33. Mocarski ES, Bonyhadif M, Salimif S, et al. (1993): Human cytomegalovirus in a SCID-hu mouse: Thymic epithelial cells are prominent targets of viral replication. Proc Natl Acad Sci 90: 104-108.

34. Lewin SR, Heller G, Zhang L, et al. (2002): Direct evidence for new T-cell generation by patients after either T-cell-depleted or unmodified allogeneic hematopoietic stem cell transplantations. Blood 100: 2235-2242.

35. Brown JA, Stevenson K, Kim HT, et al. (2010): Clearance of CMV viremia and survival after double umbilical cord blood transplantation in adults depends on reconstitution of thymopoiesis. Blood 115: 4111-4119.

36. Chaudhry MS, Velardi E, Malard F, van den Brink MRM (2017): Immune Reconstitution after Allogeneic Hematopoietic Stem Cell Transplantation: Time To T Up the Thymus. J Immunol 198: 40-46.

37. Dixit VD (2010): Thymic Fatness and Approaches to Enhance Thymopoietic Fitness in Aging. Curr Opin Immunol 22: 521528.

38. Yang H, Youm YH, Vandanmagsar B, et al. (2009): Obesity accelerates thymic aging. Blood 114: 3803-3812. 